\title{
DALL'INCHIESTA “MANI PULITE" ALLE LEGGI "AD PERSONAM". UNA RIFLESSIONE SU CLASSE POLITICA, MAGISTRATURA E CONTRASTO ALLA CORRUZIONE IN ITALIA
}

DA OPERAÇÃO "MÃOS LIMPAS" ÀS LEIS "AD PERSONAM": UMA REFLEXÃO SOBRE CLASSE POLÍTICA, MAGISTRATURA E OPOSIÇÃO À CORRUPÇÃO NA ITÁLIA

FROM THE "CLEAN HANDS" CASE TO THE "AD PERSONAM" LAWS: A STUDY ON POLITICAL CLASS, JUDICIARY AND THE FIGHT AGAINST CORRUPTION IN ITALY

DE LA OPERACIÓN "MANOS LIMPIAS" A LAS LEYES "AD PERSONAM": UNA REFLEXIÓN SOBRE LA CLASE POLIITICA, LA MAGISTRATURA Y LA OPOSICIÓN A LA CORRUPCIÓN EN ITALIA

Jacopo Paffarini ${ }^{1}$

Riassunto: Nella storia italiana l'inchiesta di Mani Pulite rappresenta un punto di svolta epocale, destinato a lasciare traccia nel rapporto tra la mag-

1 Doutor em Direito Público (Università degli Studi di Perugia/Itália). Pós-Doutor em Direito Público (Faculdade Meridional IMED). Professor Permanente no Curso de Mestrado em Direito na Faculdade Meridional IMED, Passo Fundo, RS, jacopo.paffarini@imed.edu.br. 
istratura e la classe politica negli anni a seguire. Se, da un lato, le ragioni del conflitto sono radicate nel modello di sviluppo perseguito dall'Italia per tutto il dopoguerra fino agli anni novanta, dall'altro, esiste una forte implicazione con il regime delle immunità parlamentari previsto dalla Costituzione italiana del 1948. Il presente lavoro intende ripercorre le principali vicende storiche e la cronaca giudiziaria nel tentativo di offrire uno spunto di riflessione per il Brasile travolto dall'inchiesta Lava-Jato.

Parole chiave: Mani Pulite; Immunità parlamentari; Corruzione politica; Legittimità costituzionale e principio di legalità.

Resumo: Na história italiana, a investigação "Mãos Limpas" representa uma importante virada histórica, uma vez que modifica as relações entre o Judiciário e a classe política nos anos seguintes. Se por um lado as razões do conflito estão enraizadas no modelo de desenvolvimento percorrido na Itália durante todo o período pós-guerra até os anos noventa, por outro lado, há uma forte implicação com o regime da imunidade parlamentar previsto na Constituição italiana de 1948. O presente trabalho pretende relembrar os principais acontecimentos históricos e a crônica judiciária na tentativa de fornecer elementos de reflexão para um Brasil abalado pela operação Lava-Jato.

Palavras chave: Mãos Limpas; Imunidade Parlamentar; Corrupção Política; Legitimidade Constitucional e Princípio da Legalidade.

Abstract: The "Clean Hands" inquiry represents an essential turning point in Italian history, as it modifies the traditional relationship between the Judiciary and political class in the following years. On the one hand, the causes of the institutional conflict are rooted in the Italian development model that characterized the country from the Post war period to the nineteen nineties; on the other hand, there is a strong involvement with the regimen of parliamentary immunity established by the 1948 Italian Constitution. The present study recalls the main historical events and 
crime reports, seeking to provide elements of reflection for a Brazil shaken by the Lava-Jato investigation.

Keyword: Clean Hands; Parliamentary Immunities; Political Corruption; Constitutional Legitimacy and Principle of Legality.

Resumen: En la historia italiana la investigación "Manos Limpias" representa un importante cambio histórico, puesto que modifica las relaciones entre el Poder Judicial y la clase política en los años siguientes. Si por un lado las razones del conflicto están arraigadas en el modelo de desarrollo recorrido por Italia durante todo el período de pos-guerra hasta los años noventa, por el otro, hay una estrecha implicación con el régimen de la inmunidad parlamentaria previsto en la Constitución Italiana de 1948. El presente trabajo pretende recordar los principales acontecimientos históricos y la crónica judicial en la tentativa de proporcionar elementos de reflexión para un Brasil quebrantado por la operación Lava-Jato.

Palabras clave: Manos Limpias; Inmunidad Parlamentaria; Corrupción Política; Legitimidad Constitucional y Principio de la Legalidad.

\section{INTRODUZZIONE}

possibile rilevare una coincidenza interessante tra I'Italia e il Brasile
nel 1992: in entrambi i paesi lo scandalo corruzione travolge il sistema
politico, determinando due eventi di notevole importanza per il successivo sviluppo delle istituzioni. Le indagini del Pubblico Ministero di Milano si estendono in breve tempo ai maggiori partiti politici italiani e all'intero territorio nazionale, accompagnate da una fortissima mobilitazione della società civile che continua fino al crollo elettorale delle formazioni storicamente alla guida del paese nelle elezioni del 1994. Dall'altra parte del mondo, l'impeachment del Presidente Fernando Collor de Mello immerge nello scandalo corruzione il primo Capo di Stato brasiliano eletto direttamente dal corpo elettorale dopo trent'anni di dittatura militare, sollevando l'indignazione popolare, espressa dal movimento "caras pintadas", contro il sistema di arrichimento illecito che passò alla storia 
come "schema PC" (dal nome del tesoriere Paulo César Farias che curò la raccolta fondi per la campagna elettorale di Collor).

Pur ritrovandoci in entrambi i casi davanti ad uno schema di «corruzione sistemica »², è tuttavia chiaro che c'è una differenza sostanziale tra quello che stava accadendo nei due paesi.

Il processo di impeachment è un'attività di indagine che si sviluppa "dentro il potere politico", secondo una procedura definita dalla classe politica, con l'obiettivo di punire condotte illecite realizzate nell'esercizio del mandato, secondo parametri e criteri di riscontro fondati sull'opportunità politica. Dall'altra parte, l'operazione "Mani Pulite" è un indagine portata avanti da un potere che entra nelle dinamiche della politica utilizzando gli strumenti e le chiavi di lettura del codice penale, i principi della legalità e dell'eguaglianza di tutti i cittadini davanti alla giustizia.

Tangentopoli è la punta di un iceberg, il culmine della crisi di una cultura politica nell'ambito della quale la relazione illecita di imprese, partiti e Amministrazione Pubblica risulta fondamentale per la sostenibilità dei governi che si avvicendano durante la Prima Repubblica italiana. È per questo che, quando la magistratura entra nel sistema di finanziamento illecito dei partiti, il risultato non è soltanto un'indagine giudiziaria, ma un "effetto-domino" che travolge tutti i settori della società italiana.

Il processo Mani Pulite apre una crisi istituzionale, perché mette in contrapposizione magistratura e politica per gli anni a seguire. Ma come è potuto accadere che un'inchiesta giudiziaria abbia assunto un carattere così incisivo e così distruttivo allo stesso tempo?

Le conseguenze dell'inchiesta "Mani Pulite" trovano due spiegazioni: una di natura politico-economica, legata alla fine di un modello di sviluppo che aveva

2 Sul concetto di «corruzione sistemica» si veda DELLA PORTA, Donatella; VANNUCCI, Alberto. Mani impunite. Vecchia e nuova corruzione in Italia. Bari-Roma: Laterza, 2007, p. 84: «La corruzione sistemica è modellata da un complesso di regole di comportamento, con ruoli ben strutturati fra diversi individui appartenenti al gruppo organizzato dei corrotti, e da relazioni di connivenza con altre strutture di controllo e agenti non direttamente coinvolti». Per un approfondimento degli schemi di funzionamento e degli agenti (istituzionali e non) della corruzione sistemica cfr. VANNUCCI, Alberto. Atlante della corruzione. Torino: Edizioni Gruppo Abele, 2012, p.44 e ss. 
caratterizzato I'Italia fino all'inizio degli anni '90, I'altra di natura giuridica, legata alla particolare estensione delle immunità parlamentari che sottraggono i membri del Legislativo dal dominio della legge e che, negli anni dell'inchiesta di Milano, divengono l'obiettivo della critica dell'opinione pubblica.

\section{IL CONTESTO DI “MANI PULITE”: LA NASCITA DELL'UNIONE EUROPEA E LA DEREGULATION DELL'ECONOMIA}

Con riferimento al primo aspetto, è indispensabile contestualizzare l'emersione di Tangentopoli nella svolta introdotta nella scena internazionale dalla globalizzazione dei mercati e della crescita del ruolo degli organismi sovranazionali nella regolazione dell'economia. Il Trattato di Maastricht del 1992, che sancisce la creazione dell'Unione Europea, significa l'avanzamento di un modello basato su vincoli comuni che dettano dei rapidi processi di modernizzazione dello Stato in funzione della libera concorrenza e della deregulation di tutti i settori economici. Si osservi che l'istituzione del mercato unico europeo è un processo che fin dall'inizio si manifesta in una forma tutt'altro che "indolore" e "spontanea" - come, tra l'altro, è in seguito emerso con la crisi dell'unione monetaria, il ritorno dei particolarismi nazionali e la crescente richiesta di democratizzazione dei processi decisionali comuni. L'Italia, come altri paesi del Vecchio Continente, ha inoltre un motivo storico che rende il rinnovamento richiesto dall'Unione Europea ancor più difficile.

Come è stato osservato da alcuni studiosi di questo periodo, tra cui Giovanni Orsina $^{3}$, si deve guardare alla storia dello Stato italiano per comprendere le ragioni per cui la sua classe politica diventa improvvisamente una presenza così ingombrante e onerosa da giustificare agli occhi dell'opinione pubblica un'azione giudiziaria così estesa e profonda come Mani Pulite.

In primo luogo, si consideri che la Pubblica Amministrazione italiana è tradizionalmente "iper-politicizzata". L'Italia è stato uno dei paesi da sviluppo tardivo in un contesto conflittuale come quello del Vecchio Continente agli inizi 3 ORSINA, Giovanni. Chiudere Tangentopoli: il ritorno alla normalità. Rivista Il Mulino, n. $3 / 2003$. 
del XX secolo, dove un basso indice di sviluppo significava non solo assumere una posizione servile rispetto agli altri paesi limitrofi, ma diventare, con ogni probabilità, un obiettivo di conquista. Per questo motivo la classe politica italiana affida allo Stato il compito di modernizzare il paese. È lo Stato che investe in vari settori dell'economia e delle infrastrutture per garantire la crescita degli affari e la distribuzione della rendita. Si tratta di una strategia amministrativa che ha assicurato una certa uniformità nello sviluppo sociale e nell'accesso ai servizi pubblici. Questa concezione delle funzioni dello Stato ha trovato una sostanziale continuità nel corso del XX secolo, con diversi risultati e dispositivi di comando a seconda delle ideologie prevalenti nel momento: inizia con il governo Giolitti continua il periodo fascista e si conclude con la costruzione dello Stato sociale nel dopoguerra. In questo periodo si rafforza la centralità dei partiti politici nella Pubblica Amministrazione ${ }^{4}$.

Nei primi anni '80 si manifesta una rottura tra la politica istituzionale (la "vecchia politica") e la cultura politica diffusa (la "nuova politica"): il ruolo dello Stato nell'economia e nella società continua a crescere (insieme alla burocrazia statale), mentre una società attraversata da un processo di profonda trasformazione inizia a denunciare la "partitocrazia" come un sistema che non è più in grado di rispondere alle richieste di un mercato sempre più transnazionale ed europeo.

"Tangentopoli" è Milano, la città di imprenditori che se vogliono vincere gare pubbliche devono pagare una tangente ai politici. Ma Milano è anche la "Milano da bere", la città della finanza, degli yuppies, dei super-ricchi che hanno fatto la loro strada nel mondo degli affari. È infine la città da cui parte una grande ondata popolare che chiede la prigione per i politici corrotti, la fine della burocrazia, la fine della pressione fiscale. Sembrava quasi una rivoluzione, all'inizio, il momento di transizione che ci ha portato alla Seconda Repubblica italiana.

All'inizio degli anni '90 i partiti incominciano a perdere rappresentatività, i mass-media diventano un punto di riferimento per i cittadini, sostituendo

4 Per una ricostruzione delle caratteristiche della Pubblica Amministrazione italiana e delle loro implicazioni nell'attuale contesto economico del paese, cfr. BALLATORE, Benedetto Francesco; FERRARA, Francesco; FILADELFIA, Bartolomeo. Le mancate scelte della politica italiana e il loro ruolo nella perdita della sovranità nazionale. In Amministrativamente. Rivista di diritto amministrativo. n. 7-9/2015. 
il giornalismo militante e organico alle formazioni politiche. Alcuni quotidiani nazionali ("La Repubblica", diretta da Eugenio Scalfari, "Il Giornale" di Indro Montanelli) si fanno portavoce di vasto movimento di opinione che appoggia un cambiamento nel modo di "fare politica" e per questo sostiene pienamente il lavoro della magistratura milanese. "La Repubblica", ad esempio, pubblica un editoriale, firmato da Ferdinando Adornato, in cui si afferma che il Parlamento, in crisi piena sovranità, non rappresenta più il paese. Le strade e le piazze d'Italia ormai decretano la mancanza di legittimità delle istituzioni tradizionali, temporaneamente sostituiti da tre nuovi "poteri" autenticamente rappresentativi del popolo: la campagna referendaria contro il finanziamento pubblico ai partiti politici, il Capo dello Stato (Oscar Luigi Scalfaro) e il Pubblico Ministero di Milano5. Luciano Carfagna, altro attento testimone di quel periodo, utilizza una metafora molto efficace per descrivere la situazione italiana dell'epoca, paragonandola ad una "grande slavina"6. Il termine "slavina", infatti, trasmette la sensazione di un collasso incontrollabile di un sistema che, fino a quel momento, aveva assicurato lo sviluppo italiano e che improvvisamente crolla perché non riesce più a sostenere le nuove esigenze della realtà economica e sociale.

È in tale contesto che trova spazio la figura politica di Silvio Berlusconi: come imprenditore nel settore della comunicazione e proprietario di importanti mass media, il "Cavaliere" sfrutta il divario tra "vecchia" e "nuova" politica per togliere legittimità ai propri avversari ${ }^{7}$. Berlusconi non solo nega la moralità dei partiti di sinistra post-comunisti, ma estende l'appellativo "comunista" a tutti coloro che ostacolano i suoi progetti di riforma. Li accusa di essere figli dei politici di professione che hanno lasciato agli italiani uno Stato inefficiente, pesante e non più in grado di rimanere nel mercato europeo.

Il discorso di Berlusconi trova spazio tra gli elettori per il suo appeal mediatico e perché si concretizza in semplici slogan. In primo luogo, rivendica che la politica dovrebbe affrontare i problemi della gente comune e che, pertanto, le questioni pubbliche dovrebbero essere affrontate secondo un approccio pragmatico e non 5 ADORNATO, Ferdinando. Attenti, rischiamo Weimar. In "La Repubblica" del 1/5/1993, consultabile al sito web del giornale: http://ricerca.repubblica.it/repubblica/archivio/ repubblica/1993/05/01/attenti-rischiamo-weimar.html

6 CAFAGNA, Luciano. La grande slavina. L'Italia verso la crisi della democrazia.Venezia: Marsilio, 1993.

7 Tra le più recenti ricostruzioni della parabola politica di Silvio Berlusconi, cfr. ORSINA, Giovanni. Il berlusconismo nella storia d'Italia. Venezia: Marsilio Editori, 2013. 
ideologico. In secondo luogo, dato che la vecchia classe politica e la sua cultura hanno fallito, il nuovo leader del centro-destra ritene che sia arrivato il tempo di chiedere agli imprenditori una partecipazione più attiva nella scena politica. Questa svolta, però, pone all'ordine del giorno il problema della riforma delle istituzioni: una "nuova politica", con uno spirito pragmatico e intraprendente, ha bisogno di un nuovo Stato, più "snello", cioè con meno burocrazia, in modo da essere adatto per nuova missione.

La storia giudiziaria di Tangentopoli si conclude nel 1994, lo stesso anno in cui Berlusconi assume per la prima volta il governo del paese. Il conflitto di interessi e i procedimenti penali del Cavaliere sono destinati così a diventare uno dei principali argomenti del confronto politico in Italia fino al 2011, contribuendo a rendere ancora più profonda la rottura tra i partiti politici, da un lato, la magistratura e la società civile, dall'altro ${ }^{8}$.

\section{L'IMMUNITÀ DEL PARLAMENTARE NELLA COSTITUZIONE E LA RIFORMA COSTITUZIONALE DEL 1993}

Non si può comprendere il conflitto tra organi dello Stato creato da Tangentopoli senza considerare la particolare estensione delle prerogative dei deputati e dei senatori nei confronti degli altri poteri costituzionali, ed in particolare di quello giudiziario. La Costituzione italiana del 1948 ha ritenuto necessario proteggere l'esercizio delle funzioni parlamentari prevedendo un ampio dispositivo di garanzie, riferito non soltanto all'ordinario svolgimento dell'incarico, ma dirette a prevenire qualsiasi impedimento di carattere fisico all'attuazione del mandato. Il primo aspetto delle immunità è identificato dal primo comma dell'art. 68, il quale dichiara che «i membri del Parlamento non possono essere chiamati a rispondere delle opinioni espresse e dei voti dati nell'esercizio delle loro funzioni». Il secondo è invece previsto nel capoverso e nel terzo comma dell'articolo, diretto a garantire l'inviolabilità della persona del parlamentare, ossia l'impossibilità di aprire un procedimento penale o di adottare di qualsiasi misura di restrizione fisica, compresa la perquisizione personale o domiciliare, senza l'autorizzazione della Camera di appartenenza.

8 STAFFEN, Márcio Ricardo. Magistrati. Revista Novos Estudos Jurídicos, Itajaí, v. 18, p. 569, 2013. 
Le immunità parlamentari fanno parte di un sistema razionalizzato di "checks and balances" che segna la diversità del costituzionalismo contemporaneo dallo Stato di diritto liberale e, dunque, comporta il superamento dell'idea che la legittimità dell'esercizio dei poteri - di qualsiasi potere dello Stato - possa coincidere con la mera legalità formale 9 . La formulazione dell'art. 68 della Costituzione trova dunque le sue ragioni nella storia italiana, negli abusi compiuti dal fascismo nei confronti dei parlamentari che tentarono di fermare l'ascesa di Mussolini. I tentativi di opposizione rimasero vani, infatti, non solo per la divisione del fronte antifascista, ma anche per l'assenza di garanzie volte a salvaguardare l'esercizio del mandato elettorale dagli abusi degli altri poteri. È opportuno ricordare, in questo senso, che la decadenza del mandato dei 123 parlamentari rifugiatisi sul Colle Aventino per protesta contro l'omicidio Matteotti viene decretata dalla maggioranza fascista in conformità all'art. 49 dello Statuto Albertino che imponeva ai deputati di esercitare il proprio mandato «col solo scopo del bene inseparabile del Re e della Patria» e da cui viene ricavata la decadenza dell'incarico dopo un periodo di assenza dalla Camera. Con la perdita della carica e il conseguente venir meno delle immunità penali, la maggior parte dei capi dell'opposizione antifascista furono costretti a rifugiarsi in esilio per scampare all'arresto dei giudici vicini al nuovo Duce. Coloro che rimasero nel paese, come il deputato comunista Antonio Gramsci, furono rinchiusi nelle prigioni del nuovo Stato fascista nel giro di pochi mesi ${ }^{10}$.

L'esperienza dell'ascesa del fascismo aveva dunque dimostrato la fragilità dello Stato di diritto quando la minaccia al principio di separazione dei poteri arriva da "dentro", non dalle insurrezioni di piazza, ma da uno dei poteri dello Stato. È per questo che l'Assemblea Costituente ha considerato un requisito essenziale per la tenuta dell'ordinamento repubblicano il fatto che i titolari delle più alte cariche pubbliche - non solo i parlamentari - vengano sottratti dal puro dominio della legge.

Quando l'inchiesta della Procura di Milano contro i finanziamenti illeciti ai partiti arriva all'apice del potere politico si trova la strada sbarrata dal sistema 9 ZAGREBELSKY, Gustavo. Le immunità parlamentari. Natura e limiti di una garanzia costituzionale. Torino: Einaudi, 1979.

10 DAL PONT, Adriano; CAROLINI Simonetta. L'Italia al confino 1926-1943. Le ordinanze di assegnazione al confino emesse dalle Commissioni provinciali dal novembre 1926 al luglio 1943, Milano: La Pietra, 1983. 
delle "autorizzazioni a procedere" che la Costituzione del 1948 aveva posto a protezione della inviolabilità del parlamentare. È il 29 aprile del 1993, giorno in cui la Camera dei Deputati nega l'autorizzazione ad aprire l'inchiesta contro Craxi. Inizia, così, la contesa tra la funzione della magistratura, garante della legalità, e l'autonomia della politica, legittimata dall'art. 68, comma 2, della Costituzione italiana a valutare le richieste dei giudici sulla procedibilità dell'azione penale contro i parlamentari.

L'Italia del 1993, tuttavia, non è più il paese arretrato del dopoguerra. Le promesse della Costituzione si sono in parte avverate, la società è notevolmente progredita e i diritti quali l'educazione e la salute pubblica, la partecipazione alla vita politica sono diventati una realtà per la grande maggioranza della popolazione. Però il "miracolo economico" italiano ha avuto un prezzo, ossia la trasformazione della democrazia in una "partitocrazia" che non ha eguali in Europa. Il sistema della rappresentanza entra in una fase degenerativa nel momento in cui le formazioni partitiche monopolizzano non solo il potere politico, ma la stessa vita politica organizzata. In questo senso, come sostenne Bobbio, i partiti vengono criticati perché, non sono più capaci di fare da tramite delle domande sociali e politiche, ma al contrario sono divenuti il diaframma, l'ostacolo alla modernizzazione delle strutture dello Stato e dell'ordinamento giuridico ${ }^{11}$.

Nel 1993, dunque, I'attacco alle fonti di sostengo della partitocrazia italiana si svolge su due fronti: da un lato, il referendum abrogativo della legge 195/1974 sul finanziamento pubblico ai partiti politici approvato il 19 aprile da 31 milioni di italiani, dall'altro il rifiuto del Presidente della Repubblica Oscar Luigi Scalfaro di firmare il Decreto Conso che depenalizzava il finanziamento illecito ai partiti perché ritenuto incostituzionale (è la prima volta nella storia italiana che un Capo di Stato si oppone alla decretazione d'urgenza di un governo).

Nello stesso anno, sotto l'onda delle pressioni popolari, viene approvata dal Parlamento la riforma delle immunità parlamentari (legge cost. del 29 ottobre 1993, n.3) che elimina la richiesta di autorizzazione a procedere alla Camera di appartenenza per l'apertura di un procedimento penale e introduce una deroga 11 Sul concetto di "partitocrazia" e I'analisi di Bobbio dei partiti degli anni novanta in Italia, cfr. MATTEUCCI, Nicola. Partitocrazia, in BOBBIO, Norberto; MATTEUCCI, Nicola; PASQUINO, Gianfranco. Dizionario di Politica. Torino: Utet, 2016, p. 691. 
all'inviolabilità della libertà fisica del parlamentare nel caso di sentenze definitive di condanna e nel caso di flagranza di reato. La riforma costituzionale è una vittoria del potere giudiziario, che conta su un ampio appoggio popolare, contro una classe politica che è costretta a cedere una parte del terreno delle immunità. Una vittoria che però la magistratura si affretta a definire "parziale" perché il parlamentare rimane ancora un soggetto privilegiato rispetto al resto della cittadinanza. Ad esempio, non può essere sottoposto all'arresto cautelare davanti al rischio di reiterazione delle condotte o di inquinamento delle prove, le sue comunicazioni non possono essere intercettate e la sua corrispondenza non è suscettibile a sequestro ${ }^{12}$. Per tutti questi provvedimenti è ancora necessario che la richiesta del Tribunale procedente sia approvata dall'assemblea di appartenenza.

\section{L'EVOLUZIONE DEL CONFLITTO TRA POLITICA E MAGISTRATURA: LE LEGGI "AD PERSONAM" AL VAGLIO DELLA CORTE COSTITUZIONALE}

Davanti a queste trasformazioni dell'assetto delle immunità la politica non tarderà a presentare la sua controffensiva. In un primo momento con il "Decreto Biondi" (dal nome del Ministro della Giustizia del Primo governo Berlusconi) che sostituisce gli arresti domiciliari alla carcerazione nella fase istruttoria dei procedimenti per corruzione. Il provvedimento viene tuttavia ritirato, dopo le proteste popolari provocate dalle immagini, divulgate dai giornali il giorno dopo la sua emanazione, raffiguranti politici e imprenditori sotto indagine mentre escono di prigione. In un secondo momento, ritorna al centro il tema delle immunità con la vicenda del "Lodo Schifani". Sono passati diversi anni dall'inchiesta di Tangentopoli (siamo nel 2003), ma il sistema di finanziamento illecito che lega il mondo dell'imprenditoria alla politica è ancora in piedi. La legge 140/2003 (Disposizioni per l'attuazione dell'articolo 68 della Costituzione nonché in materia di processi penali nei confronti delle alte cariche dello Stato) introduce all'art. 1 una garanzia di improcedibilità nei confronti delle cinque più alte cariche dello Stato, sfruttando gli spazi di interpretazione lasciati aperti dalla riforma dell'art. 68 della Costituzione. Non potendo più impedire l'inizio del procedimento penale, 12 MONTAGNA, Mariangela. Le autorizzazioni ad acta per i parlamentari. In In ANGELINI, MarCo; OLIVIERO, Maurizio. Le immunità nel diritto interno e comparato. Atti del convegno di Perugia 25-27 maggio 2006, Torino: Giappichelli, 2014. 
la maggioranza parlamentare che sostiene il II governo Berlusconi sancisce la temporanea sospensione dei processi per tutti i reati, anche anteriori all'assunzione della carica, del Presidente della Repubblica, Presidente del Senato, Presidente della Camera, Presidente del Consiglio dei Ministri e del Presidente della Corte Costituzionale. Si noti che l'unico soggetto ad essere stato rinviato a giudizio in più occasioni al momento dell'entrata in vigore del decreto era il Premier Berlusconi e che la sua strategia processuale si è basata esclusivamente sull'estinzione dei reati per la prescrizione dei termini di celebrazione del giudizio.

Il lodo Schifani cade perché dichiarato illegittimo dalla Corte Costituzionale (sent. 24/2004), la quale conquista il titolo di "custode della costituzione", spiazzando il Presidente della Repubblica dal quale l'opinione pubblica si aspettava un nuovo "veto", come fece Scalfari con il Decreto Conso. Si tratta tuttavia di un posizionamento ambiguo da parte dei giudici: la Corte dichiara incostituzionale la norma, non in quanto la stessa introduce surrettiziamente una "nuova" immunità, ma perché non giustifica la disparità di trattamento accordata ai Presidenti degli organi costituzionali rispetto agli altri membri che li compongono. In altre parole, se l'improcedibilità vale per il Presidente del Consiglio, deve valere, in base ad un criterio di uguaglianza, anche per i singoli Ministri, nella misura in cui anch'essi svolgono una funzione costituzionale. È evidente che si è trattato di un "self restraint" della Corte, la quale non dice se ampliare le immunità è costituzionalmente legittimo o illegittimo, ma solo che non può essere fatto creando disparità all'interno dei componenti degli organi costituzionali.

La decisione, in tal forma, posticipa solo il problema. Alcuni anni più tardi, infatti, una nuova legge ad personam, il Lodo Alfano, promossa dal Ministro della Giustizia del IV governo Berlusconi, ripropone lo stesso schema con piccole modifiche (come la possibilità di rinunciare alla condizione di improcedibilità, la limitazione della durata dell'improcedibilità alla durata del mandato, I'esperibilità delle prove non rinviabili, la tutela della parte civile del processo). La Corte Costituzionale questa volta respinge la legge (sentenza n. 262/2009) contestando, finalmente, I'introduzione con norma ordinaria di un privilegio sostanzialmente equivalente alle immunità costituzionalmente sancite. Nella misura in cui l'Assemblea Costituente ha deciso di collocare la disciplina delle immunità nel testo della Costituzione, il 
loro contenuto può essere oggetto di modifica solamente attraverso la revisione del testo della Carta ex art. 138 Cost.

Di nuovo, però, occorre rilevare che bocciando il Lodo Alfano la Corte non si schiera contro le "ragioni della politica", come alcuni giornalisti hanno dichiarato tentando di far passare i giudici come degli "attivisti" nella lotta contro la corruzione politica. Al contrario, la Corte Costituzionale italiana afferma che l'arbitro dei conflitti tra poteri rimane ancora il testo della Costituzione, non il codice penale. Più precisamente, che esiste una sfera della legalità e una sfera della legittimità e che le immunità appartengono a quest'ultima e pertanto solo con un procedimento di revisione costituzionale si può ridisegnare una tale materia ${ }^{13}$.

È chiaro che le immunità introducono una deroga al principio di legalità e sono un'eccezione allo Stato di diritto (nel quale tutti i cittadini sono uguali davanti alla legge $)^{14}$. È inoltre evidente il motivo della loro "impopolarità", dal momento che proteggono (solo) alcuni soggetti dalle conseguenze di condotte illecite, i quali, per questo motivo, sono dipinti come una "casta" dagli organi di informazione. Tuttavia, le immunità parlamentari sono state accordate ai titolari del potere politico, non soltanto perché la loro funzione trova legittimazione nella democrazia elettorale, ma perché è al Parlamento e al Governo che l'Assemblea Costituente ha affidato il compito di dare attuazione principi fondamentali della Repubblica nata dalla Resistenza antifascista e, in particolare, ai diritti fondamentali proclamati nel testo della Costituzione.

\section{ALCUNE RIFLESSIONI FINALI}

Non mancano profili critici nell'ultima decisione della Corte Costituzionale a proposito del Lodo Alfano: di nuovo non si chiarisce quale sia il "legittimo equilibrio" nei rapporti tra magistratura e politica ${ }^{15}$.

13 GIUPPONI, Tommaso Francesco. La sentenza sul "lodo Alfano": le possibili prospettive di riforma. In Quaderni costituzionali. n. 4/2009, p. 929-932. Per una diversa opinione cfr. CARNEVALE, Paolo. "A futura memoria": dalla Corte segnali "per il dopo". In A. CELOTTO, Prerogativa o privilegio? Opinioni a confronto sul lodo Alfano, Roma, 2009.

14 Sul punto cfr. GIUPPONI, Tommaso Francesco. Le immunità costituzionali. In ANGELINI, Marco; OLIVIERO, Maurizio. Le immunità nel diritto interno e comparato. Atti del convegno di Perugia 25-27 maggio 2006, Torino: Giappichelli, 2014.

15 ALVES, Fernando de Brito; BREGA FILHO, Vladimir. Termidorizar a deliberação: o papel das cortes constitucionais nas democracias contemporâneas. Revista Brasileira de Direito, [S.I.], v. 11, n. 1, p. 124-134, ago. 2015. 
Quali limiti incontra una eventuale estensione delle immunità fatta con legge costituzionale? Può essere legittimo un "Lodo Alfano costituzionale", ossia una riforma del testo della Carta fondamentale che introduce la sospensione dei processi ritardando l'opera degli organi di giustizia o, peggio, garantendo una sostanziale impunità per i membri del Parlamento? Quando è possibile parlare di una lesione delle finalità costituzionali del potere giudiziario?

Il problema è ancor più rilevante se si considera che l'attuale sistema elettorale italiano ha un effetto fortemente maggioritario e può dare ad una coalizione uscita vincente dalle elezioni i seggi parlamentari sufficienti per procedere ad una revisione della Carta fondamentale ${ }^{16}$. In tal senso, è stata giustamente richiamata la necessità di una riforma della stessa procedura di revisione costituzionale aumentando la maggioranza necessaria per l'approvazione degli emendamenti (attualmente 2/3 dei componenti di ciascuna Camera). Ma l'appello dei costituzionalisti è caduto nel vuoto ${ }^{17}$.

Dopo diversi anni questa prospettiva è in procinto di avverarsi: nel 12 aprile scorso il governo di Renzi ha approvato una riforma costituzionale, che sarà sottoposta a referendum entro la fine dell'anno. Il testo approvato dal Parlamento prevede una modifica della composizione e delle funzioni del Parlamento, ma non ha ridotto la portata delle immunità. Al contrario, ha esteso l'inviolabilità penale dei deputati ai membri del nuovo Senato che darà rappresentanza alle istituzioni territoriali ${ }^{18}$.

Un secondo problema riguarda lo scenario costituzionale che si aprirebbe nel caso in cui il rifiuto parlamentare di una richiesta di autorizzazione a procedere 16 Il sistema elettorale vigente (legge 6 maggio 2015, n. 52) prevede l'assegnazione dei seggi su base proporzionale, con uno sbarramento del 3\% e un premio di maggioranza per la lista vincitrice pari al $54 \%$ dei seggi nella Camera dei Deputati. Il premio è attribuito all'esito del primo turno, se la lista più votata ha raggiunto il $40 \%$ dei suffragi, altrimenti alla lista vincitrice del ballottaggio che si deve svolgere tra le due liste più votate nel primo turno. La formula elettorale attuale si pone in sostanziale continuità con la precedente (legge 21 dicembre 2005, n. 270), con la quale è stato l'attuale Parlamento italiano e che è stata dichiarata incostituzionale proprio in riferimento al premio di maggioranza e all'impossibilità per gli elettori di esprimere una preferenza tra i candidati della lista. Sul punto si veda la sentenza della Corte Costituzionale italiana n. 1/2014.

17 LANCHESTER, Fulco. Contributo per il discernimento costituzionale. In Osservatorio costituzionale, n.1, 2016, disponibile al sito web: http://www.osservatorioaic.it/un-contributoper-il-discernimento-costituzionale-cd8.html

18 Il testo della riforma costituzionale è disponibile presso il sito della Camera dei Deputati: http://documenti.camera.it/leg17/dossier/pdf/ac0500n.pdf 
venga impugnata da parte della magistratura procedente. La Corte Costituzionale, in tal caso, non avrebbe solidi appigli normativi per giudicare un conflitto di attribuzioni di questo genere. L'unico riferimento è fornito dall'art. 9 della legge costituzionale n. 1 del 1989 che, rispetto alle autorizzazioni a procedere contro i membri del Governo, afferma che la Camera di appartenenza «può, a maggioranza assoluta dei suoi componenti, negare l'autorizzazione a procedere ove reputi, con valutazione insindacabile, che l'inquisito abbia agito per la tutela di un interesse dello Stato costituzionalmente rilevante ovvero per il perseguimento di un preminente interesse pubblico nell'esercizio della funzione di Governo» [corsivo dell'Autore]. Tuttavia, è chiaro che la formula della legge costituzionale è molto vaga e non risolve il problema della discrezionalità politica di cui è già provvista l'assemblea nel valutare le richieste della magistratura.

In conclusione, è opportuno ricordare che nel diritto costituzionale italiano la magistratura esercita le sue funzioni in nome del popolo italiano, nella misura in cui, come afferma l'articolo 101, comma 1, della Costituzione «la giustizia è amministrata in nome del popolo». Per non essendo investita da un mandato elettore, dunque, la magistratura rappresenta la sovranità popolare, al lato degli organi politici, secondo le forme della rappresentanza istituzionale ${ }^{19}$. I tentativi di protagonismo personale e le tentazioni populiste, di entrambe le parti, sono pertanto da ritenersi estranei allo spirito del costituzionalismo contemporaneo, anche quando trovano fondamento nella lotta alla corruzione politica.

Ricordando le parole di Carlo Esposito ${ }^{20}$ :

"disposizione che il popolo è sovrano nelle forme e nei limiti della Costituzione non significa che la Costituzione sopravvenga per porre limiti estrinseci all'esercizio di una preesistente sovranità del popolo (e che in sostanza la Costituzione neghi la sovranità popolare per affermare la propria), ma proprio all'opposto che la sovranità del popolo esiste solo nei limiti e nelle forme in cui la Costituzione la organizza, la riconosce e la rende possibile, e fin quando sia esercitata nelle forme e nei limiti del diritto. Fuori della Costituzione e del diritto non c'è la sovranità, ma l'arbitrio popolare, non c'è il popolo sovrano, ma la massa con le sue passioni e con la sua debolezza"

19 SILVESTRI, Gaetano. Sovranità popolare e magistratura. In Costituzionalismo.it, Fascicolo 3/2003 disponibile al sito http://www.costituzionalismo.it/articoli/107/.

20 ESPOSITO, Carlo. Commento all'art. 1 della Costituzione (1948), ripubblicato in La Costituzione italiana - Saggi, Padova: Cedam, 1954, p. 11. 


\section{BIBLIOGRAFIA}

ADORNATO, Ferdinando. Attenti, rischiamo Weimar. In "La Repubblica" del 1/5/1993, consultabile al sito web del giornale: http://ricerca.repubblica.it/repubblica/archivio/ repubblica/1993/05/01/attenti-rischiamo-weimar.html

ALVES, Fernando de Brito; BREGA FILHO, Vladimir. Termidorizar a deliberação: o papel das cortes constitucionais nas democracias contemporâneas. Revista Brasileira de Direito, [S.I.], v. 11, n. 1, p. 124-134, ago. 2015.

BALLATORE, Benedetto Francesco; FERRARA, Francesco; FILADELFIA, Bartolomeo. Le mancate scelte della politica italiana e il loro ruolo nella perdita della sovranità nazionale. In Amministrativamente. Rivista di diritto amministrativo. n. 7-9/2015.

CAFAGNA, Luciano. La grande slavina. L'Italia verso la crisi della democrazia.Venezia: Marsilio, 1993.

DAL PONT, Adriano; CAROLINI Simonetta. L'Italia al confino 1926-1943. Le ordinanze di assegnazione al confino emesse dalle Commissioni provinciali dal novembre 1926 al luglio 1943, Milano: La Pietra, 1983.

DELLA PORTA, Donatella; VANNUCCI, Alberto. Mani impunite. Vecchia e nuova corruzione in Italia. Bari-Roma: Laterza, 2007

ESPOSITO, Carlo. Commento all'art. 1 della Costituzione (1948), ripubblicato in La Costituzione italiana - Saggi, Padova: Cedam, 1954.

GIUPPONI, Tommaso Francesco. Le immunità costituzionali. In ANGELINI, Marco; OLIVIERO, Maurizio. Le immunità nel diritto interno e comparato. Atti del convegno di Perugia 25-27 maggio 2006, Torino: Giappichelli, 2014.

GIUPPONI, Tommaso Francesco. La sentenza sul "lodo Alfano": le possibili prospettive di riforma. In Quaderni costituzionali. n. 4/2009, p. 929-932. Per una diversa opinione cfr. CARNEVALE, Paolo. "A futura memoria": dalla Corte segnali "per il dopo". In A. CELOTTO, Prerogativa o privilegio? Opinioni a confronto sul lodo Alfano, Roma, 2009.

LANCHESTER, Fulco. Contributo per il discernimento costituzionale. In Osservatorio costituzionale, n.1, 2016, disponibile al sito web: http://www.osservatorioaic.it/un-contributoper-il-discernimento-costituzionale-cd8.html. 
MATTEUCCI, Nicola. Partitocrazia, in BOBBIO, Norberto; MATTEUCCI, Nicola; PASQUINO, Gianfranco. Dizionario di Politica. Torino: Utet, 2016, p. 691.

MONTAGNA, Mariangela. Le autorizzazioni ad acta per i parlamentari. In In ANGELINI, Marco; OLIVIERO, Maurizio. Le immunità nel diritto interno e comparato. Atti del convegno di Perugia 25-27 maggio 2006, Torino: Giappichelli, 2014.

ORSINA, Giovanni. Il berlusconismo nella storia d'Italia. Venezia: Marsilio Editori, 2013

ORSINA, Giovanni. Chiudere Tangentopoli: il ritorno alla normalità. Rivista Il Mulino, n.3/2003.

SILVESTRI, Gaetano. Sovranità popolare e magistratura. In Costituzionalismo.it, Fascicolo 3/2003 disponibile al sito http://www.costituzionalismo.it/articoli/107/.

STAFFEN, Márcio Ricardo. Magistrati. Revista Novos Estudos Jurídicos, Itajaí, v. 18, p. 569, 2013.

VANNUCCI, Alberto. Atlante della corruzione. Torino: Edizioni Gruppo Abele, 2012.

ZAGREBELSKY, Gustavo. Le immunità parlamentari. Natura e limiti di una garanzia costituzionale. Torino: Einaudi, 1979.

Recebido em: set/2016

Aprovado em: jan/2017 